\title{
Verbal Learning Strategy Following Mild Traumatic Brain Injury
}

\author{
Elizabeth K. Geary, ${ }^{1,2}$ Marilyn F. Kraus, ${ }^{1,3,4}$ Leah H. Rubin,,${ }^{3,4}$ Neil H. Pliskin, ${ }^{1,3,4}$ \\ AND Deborah M. Little e $^{1,2,5,6,7}$ \\ ${ }^{1}$ Department of Neurology, The University of Illinois College of Medicine, Chicago, Illinois \\ ${ }^{2}$ Center for Stroke Research, The University of Illinois College of Medicine, Chicago, Illinois \\ ${ }^{3}$ Department of Psychiatry, The University of Illinois College of Medicine, Chicago, Illinois \\ ${ }^{4}$ Center for Cognitive Medicine, The University of Illinois College of Medicine, Chicago, Illinois \\ ${ }^{5}$ Department of Anatomy, The University of Illinois College of Medicine, Chicago, Illinois \\ ${ }^{6}$ Department of Ophthalmology, The University of Illinois College of Medicine, Chicago, Illinois \\ ${ }^{7}$ Department of Psychology, The University of Illinois College of Medicine, Chicago, Illinois
}

(Received December 7, 2010; Final Revision March 25, 2011; AcCePted March 28, 2011)

\begin{abstract}
That learning and memory deficits persist many years following mild traumatic brain injury (mTBI) is controversial due to inconsistent objective evidence supporting subjective complaints. Our prior work demonstrated significant reductions in performance on the initial trial of a verbal learning task and overall slower rate of learning in well-motivated mTBI participants relative to demographically matched controls. In our previous work, we speculated that differences in strategy use could explain the differences in rate of learning. The current study serves to test this hypothesis by examining strategy use on the California Verbal Learning Test-Second Edition. Our present findings support the primary hypothesis that mTBI participants under-utilize semantic clustering strategies during list-learning relative to control participants. Despite achieving comparable total learning scores, we posit that the persisting learning and memory difficulties reported by some mTBI patients may be related to reduced usage of efficient internally driven strategies that facilitate learning. Given that strategy training has demonstrated improvements in learning and memory in educational and occupational settings, we offer that these findings have translational value in offering an additional approach in remediation of learning and memory complaints reported by some following mTBI. (JINS, 2011, 17, 709-719)
\end{abstract}

Keywords: Post-concussive syndrome, Concussion, Semantic, Cognition, Executive functions, Brain/behavior relationships

\section{INTRODUCTION}

That learning and memory difficulties are an acute consequence of mild traumatic brain injury (mTBI) is well supported. That deficits persist years following injury, however, is a controversial issue. While the majority of individuals do not appear to experience persisting cognitive difficulties after mTBI, a subset of patients do demonstrate such difficulties (Benedictus, Spikman, \& van der Naalt, 2010; Ponsford et al., 2000). For a myriad of complex reasons (e.g., psychological, motivational), this subset proves a challenge for clinicians. Prior work conducted in our laboratory using a non-clinical, non-litigating sample of mTBI patients attempted to address issues related to memory complaints often raised by clinical

Correspondence and reprint requests to: Deborah M. Little, Department of Neurology, MC 796, 912 South Wood Street 855 N., Chicago, IL 60612. E-mail: deborahmlittle@gmail.com patients and their families (Geary, Kraus, Pliskin, \& Little, 2010). Our previous work focused on trial-by-trial performance on a measure of verbal learning in a sample of community-recruited mTBI participants. We reported that mTBI participants demonstrated diminished acquisition on the initial learning trial and evidenced an overall slower rate of learning across trials in the context of equivalent performance relative to controls on the total learning and memory indices (Geary et al., 2010). Furthermore, performance on the verbal learning task was related to imaging measures showing a relationship between the effects of injury on cerebral white matter integrity and behavioral performance. One limitation of our previous work was that we were unable to comment on the specific mechanism that may underlie our behavioral findings. In this previous work, we proposed the hypothesis that meta-cognitive strategy use might underlie the verbal learning deficiency in mTBI. 
Examining higher-order "meta-cognitive" learning and memory strategies has particular relevance in patient populations including mTBI where evidence of chronic primary temporal lobe/diencephalic memory dysfunction is not generally supported (Binder, Rohling, \& Larrabee, 1997; Cicerone \& Kalmar, 1995; Dikmen et al., 2009; Ettenhofer \& Abeles, 2009; Iverson, 2005; Schretlen \& Shapiro, 2003; West, Curtis, Greve, \& Bianchini, 2010). Others have argued that memory deficiencies following mTBI could be influenced by dysfunction in frontal-subcortical networks which may support metacognitive functions (Alexander, Stuss, \& Gillingham, 2009; Bruce \& Echemendia, 2003; Little et al., 2010). In learning and memory, meta-cognitive functions such as restructuring information via the identification of shared relationships between items and/or other internally driven mnemonic devices increase one's ability to learn and recall information (Becker \& Lim, 2003; Schefft, Dulay, \& Fargo, 2008). Studies in TBI and other neurologic populations provide evidence that successful recall of items on list-learning tasks is influenced by how well one consistently uses an efficient (i.e., semantic, subjective) recall strategy (Bruce \& Echemendia, 2003; Chan et al., 2000; Gongvatana et al., 2007; Gsottschneider et al., 2010; Luek, 1976; Ribeiro, Guerreiro, \& De Mendonça, 2007).

When conceptualizing meta-cognitive strategies hierarchically in terms of degree of cognitive engagement, semantic clustering arguably constitutes a sophisticated strategy. Semantic clustering encompasses mentally grouping items from the same taxonomic category at greater than chance levels and is most often associated with improved learning and recall (Delis, Freeland, Kramer, \& Kaplan, 1988). In order for semantic clustering strategies to be used, an individual must first identify that semantic relationships exist, use the strategy by compartmentalizing words during list encoding, and then use the semantic groups during both initial and subsequent recall. In list-learning tasks such as the California Verbal Learning Test (CVLT-II), this process involves recognizing that the pseudo-random presentation of 16 target words consists of items from four semantic categories, regrouping words according to these categories, and organizing these words by category during recall.

In contrast to semantic clustering, subjective clustering may involve restructuring the list based on phonemic features of items or another personally derived mnemonic strategy. Because subjective clustering is internally derived, it is suspected when one recalls two or more words together from one trial to the next independent of semantic or serial clustering strategies.

Finally, serial clustering, or recalling words in the order of presentation, may partially reflect the tendency to recall the first words and last words presented (primacy/recency effects). Of all three strategies, serial clustering requires the least amount of cognitive engagement as the structure is externally facilitated by presentation order. If used at the exclusion of the other two strategies, serial clustering tends to be the least efficient as it often results in poorer performance (Delis et al., 1988). Serial clustering is often most readily applied across trials in memory impaired populations
(Gsottschneider et al., 2010; Jefferies, Hoffman, Jones, \& Ralph, 2008; Ranjith, Mathuranath, Sharma, \& Alexander, 2010).

In our prior work, while there were no significant group differences on the traditional executive function measures in our analyses (Geary et al., 2010; Kraus et al., 2007), we speculated that differences in the rate of learning between groups could be related to less often analyzed executive functions including strategy use on the CVLT-II. Like others, we reasoned that these individualized measures of performance may be more sensitive to subtle diffuse effects following mTBI (Cicerone, Levin, Malec, Stuss, \& Whyte, 2006; Schweizer, Alexander, Gillingham, Cusimano, \& Stuss, 2010). The purpose of the present investigation is to test the hypothesis that semantic clustering will predict learning rate for control participants but not for our mTBI participants.

\section{METHODS}

\section{Participants}

From a larger sample of participants described previously (Geary et al., 2010), CVLT-II response data were available and analyzed for a total of 35 mTBI participants (19 females) and 28 healthy controls (15 females). Participants were recruited via advertisements in the community seeking individuals who had ever sustained a closed head injury, concussion, or traumatic brain injury. No participants were recruited from active clinical practices for treatment of TBI. All participants provided written informed consent and experimental procedures complied with the code of ethics of the World Medical Association, Declaration of Helsinki, and Institutional Review Board. Participants were excluded if they had a history of psychiatric disorder before the TBI, substance abuse/dependency, current or past litigation, failure on a formal measure of effort, or any other neurologic or medical condition that could result in cognitive changes (e.g., hypertension, severe chronic pain). For this study, participants were also excluded if there was positive radiologic finding of contusion or bleed, or, upon review of both T2- and T1-weighted magnetic resonance imaging, evidence of skull fracture suggesting significant trauma to the head. No mTBI participants had evidence of focal neurological symptom at the time of evaluation. Additionally, participants were not receiving any psychiatric medication or medications used for cognitive enhancement at the time of the study. The criteria used for defining mTBI follow the guidelines set forth by the American Congress of Rehabilitation Medicine (ACRM, 1993), including endorsement of at least one of the following: any period of loss of consciousness (LOC); any loss of memory for events immediately before or after the accident (PTA); any alteration in mental state at the time of the accident; focal neurological deficit (ACRM, 1993; Cassidy et al., 2004). These criteria help ensure that our sample were, in fact, mild severity (LOC less than $30 \mathrm{~min}$; PTA less than $24 \mathrm{hr}$, and/or the Glasgow Coma Scale greater than or equal to 13) (ACRM, 1993; Cassidy et al., 2004; Levin, 1992; Tagliaferri, Compagnone, Korsic, Servadei, \& Kraus, 2006). 
Table 1. Demographics and brain injury variables

\begin{tabular}{|c|c|c|c|c|c|c|}
\hline & \multicolumn{2}{|c|}{ Control $(n=28)$} & \multicolumn{2}{|c|}{$\operatorname{mTBI}(n=35)$} & \multirow{2}{*}{$t$ value } & \multirow{2}{*}{$p$ value } \\
\hline & Mean & $S D$ & Mean & $S D$ & & \\
\hline \multicolumn{7}{|l|}{ Demographic variables } \\
\hline Age & 31.64 & 9.02 & 33.91 & 10.09 & -0.93 & 0.356 \\
\hline Years of education & 15.79 & 1.73 & 16.37 & 2.09 & -1.193 & 0.238 \\
\hline Years of employment & 12.05 & 9.86 & 16.24 & 10.25 & -1.618 & 0.111 \\
\hline Hollingshead highest level of employment & 6.47 & 1.61 & 6.43 & 1.52 & 0.088 & 0.930 \\
\hline WTAR Full-Scale IQ estimate & 110.21 & 11.40 & 110.54 & 9.67 & -0.124 & 0.902 \\
\hline TOMM Trial 2 & 50.00 & 0.00 & 49.90 & 0.32 & 1.547 & 0.129 \\
\hline Dot Counting & 8.60 & 2.50 & 9.07 & 2.49 & -0.647 & 0.521 \\
\hline Employed/student at evaluation (\% sample) & \multicolumn{2}{|c|}{$92.90 \%$} & \multicolumn{2}{|c|}{$94.30 \%$} & & \\
\hline Gender $(\mathrm{M} / \mathrm{F})$ & 13 & 15 & 16 & 19 & & \\
\hline \multicolumn{7}{|l|}{ TBI variables } \\
\hline Age at TBI (years) & - & - & 28.54 & 10.81 & & \\
\hline Time since injury (years) & - & - & 5.63 & 6.57 & & \\
\hline Length of loss of consciousness $(N=17)$ (minutes) & - & - & 5.71 & 9.21 & & \\
\hline Length of post-traumatic amnesia $(N=10)$ (minutes) & - & - & 33.50 & 26.98 & & \\
\hline Returned to work/school following injury (\% sample) & - & - & \multicolumn{2}{|c|}{$94.30 \%$} & & \\
\hline
\end{tabular}

For individuals who had witness-confirmed information on duration of LOC and/or PTA the average reported LOC was 5.7 minutes $(\mathrm{N}=17$; range $=0-30 \mathrm{~min})$ and average reported PTA was $33.5 \mathrm{~min}(\mathrm{~N}=10$; range $=0-60 \mathrm{~min})$. For patients without specific information regarding LOC $(\mathrm{N}=18)$ or PTA $(\mathrm{N}=25)$, we relied upon estimates of self-report and witness report of duration of LOC or PTA. These criteria reduce the risk of Type I error as the reliance on self-report and inclusion of no minimum LOC raises the possibility that participants classified as mTBI may not have sustained a brain injury. We adopted this more conservative approach to ensure that we did not bias data in favor of the study hypothesis by including complicated mild or moderate TBI.

The mechanism of injury for the mTBI participants included motor vehicle accidents (MVA; $\mathrm{N}=9$ ), pedestrian versus MVA $(\mathrm{N}=2)$, assault $(\mathrm{N}=3)$, sports-related $(\mathrm{N}=10)$, and falls or blows to the head $(\mathrm{N}=11)$. Twelve patients reported experiencing more than one mTBI (range, 2-7 mTBI). Given that the purpose of this study was to elaborate on findings from the originally published work, and the original findings were supported regardless of the inclusion of multiple TBI patients, we did not exclude on the basis of history of multiple mTBI. Demographic data and injury related variables are presented in Table 1.

\section{MATERIALS AND PROCEDURE}

\section{Neuropsychological Assessment}

As detailed previously, participants completed an extensive neuropsychological test battery that was assembled to assess executive function, attention, and memory (Kraus et al., 2007). Performance on individual measures from this battery for both groups are presented in Table 2. The CVLT-II was used to assess list-learning and memory. In addition to capturing the amount of verbal information an individual can learn and recall, the CVLT-II measures many individualized elements of precisely how information is learned (Delis, Kramer, Kaplan, \& Obers, 2000a). We examined the following CVLT-II strategies.

\section{Calculation of Clustering Scores}

\section{Chance adjusted (CA) semantic category clustering individual trials}

Semantic clustering involves recalling two or more words by virtue of shared semantic category. Recent theories of semantic clustering argue that organization processes occur during list-learning, presumably as semantic categories are identified. Semantic cluster scores were calculated based on the list-based measure of observed minus expected clustering offered by Stricker, Brown, Wixted, Baldo, and Delis (2002), which was recently demonstrated to show improved classification rates when used with clinical samples (Delis et al., 2010). For scoring observed semantic clusters, one point is given for each correct semantic cluster (i.e., each pair of words from the same semantic category), for a maximum of 12 points for each trial. For example, successive recall of the words cat/dog/fish would yield an observed semantic cluster score of two. The CA semantic clustering score used in analyses is the observed semantic clustering score minus the expected semantic clustering score. To calculate expected semantic clustering score, we adopted the method illustrated in Equation 1 (Delis, Kramer, Kaplan, \& Obers, 2000b).

$$
\text { Eq. 1. Expected Sem Cli }=\frac{[(r-1)(m-1)]}{\mathrm{N}_{\mathrm{L}}-1}
$$

where, "i" represents a given trial, " $r$ " the number of correct words recalled on trial $i$, "m" represents the number of members 
Table 2. Neuropsychological test performance

\begin{tabular}{|c|c|c|c|c|c|c|c|}
\hline & \multicolumn{2}{|c|}{ Control $(n=28)$} & \multicolumn{2}{|c|}{$\operatorname{mTBI}(n=35)$} & \multirow{2}{*}{$t$ value } & \multirow{2}{*}{$p$ value } & \multirow{2}{*}{$\eta^{2}$} \\
\hline & Mean & $S D$ & Mean & $S D$ & & & \\
\hline \multicolumn{8}{|l|}{ Executive } \\
\hline COWAT Total & 42.79 & 11.39 & 40.51 & 11.09 & 0.798 & 0.425 & 0.010 \\
\hline CPT Errors of Commission & 11.21 & 6.27 & 14.09 & 6.55 & -1.753 & 0.085 & 0.049 \\
\hline Digit Span Backward & 8.61 & 2.42 & 7.60 & 2.66 & 1.553 & 0.126 & 0.038 \\
\hline Trails B (s) & 51.18 & 12.87 & 48.00 & 11.49 & 1.034 & 0.305 & 0.017 \\
\hline Stroop Color-Word (s) & 52.54 & 10.67 & 49.86 & 9.68 & 1.043 & 0.301 & 0.018 \\
\hline Spatial Span Backward (s) & 10.93 & 2.57 & 11.37 & 2.60 & -0.675 & 0.502 & 0.007 \\
\hline RUFF Unique Designs (s) & 45.99 & 13.43 & 43.94 & 9.03 & 0.723 & 0.472 & 0.009 \\
\hline \multicolumn{8}{|l|}{ Attention } \\
\hline Digit Span Forward (s) & 11.11 & 2.63 & 11.43 & 2.19 & -0.530 & 0.598 & 0.005 \\
\hline Spatial Span Forward (s) & 11.43 & 3.10 & 10.09 & 3.45 & 1.606 & 0.113 & 0.041 \\
\hline Trails A (s) & 51.61 & 15.21 & 48.34 & 11.12 & 0.983 & 0.329 & 0.016 \\
\hline CPT Number of Omissions Raw & 3.25 & 6.73 & 1.71 & 2.37 & 1.250 & 0.216 & 0.025 \\
\hline \multicolumn{8}{|l|}{ Other Memory } \\
\hline BVMT Trials $1-3$ Total & 27.39 & 5.00 & 25.17 & 5.23 & 1.709 & 0.093 & 0.046 \\
\hline BVMT Delay Recall & 9.96 & 1.53 & 9.49 & 1.79 & 1.125 & 0.265 & 0.020 \\
\hline
\end{tabular}

Note. $(\mathrm{s})=$ standard score; $\mathrm{CPT}=$ Conners Continuous Performance Test; COWAT $=$ Controlled Oral Word Association Test; RUFF $=$ Ruff Figural Fluency Test; BVMT $=$ Brief Visual Spatial Memory Test.

of each semantic category on the original list, and " $\mathrm{N}_{\mathrm{L}}$ " the total number of words on the original list. As such, the CA scores can range from a high of 9.0 (perfect semantic clustering with a total recall score of 16) to a low of -3.0 (no observed semantic clustering with a total recall score of 16) (Delis et al., 2000b).

\section{Chance adjusted (CA) subjective clustering individual trials}

Subjective clustering involves word pairs recalled together from one trial to the next, which do not adhere to semantic or serial clustering strategies. For example, subjective clusters may consist of seemingly unrelated words, which have been grouped using some mnemonic by the individual (e.g., car full of lettuce) or words that share phonemic qualities (e.g., sofa/soup). The observed directional subjective clustering score includes any target words recalled together (either in forward order or backward order) across two consecutive trials. The expected subjective clustering score is calculated using the method illustrated in Equation 2.

$$
\text { Eq. 2. Expected Subj Clii }=\frac{[(2 c)(c-1)]}{\mathrm{hk}}
$$

The expected value consists of "ii" which represents the subjective clustering score between two given trials, "c," which is the number of common items recalled in Trials $t$ and $\mathrm{t}+1$ (regardless if grouped together), "h," which is the number of recalled items in Trial $t$, and " $k$," which is the number of items recalled in Trial $\mathrm{t}+1$ (Sternberg \& Tulving, 1977). The CA subjective clustering score used in analyses is the observed subjective clustering score minus the expected subjective clustering score. An example is if the word pair car/lettuce (subjective observed score of 1 ) is recalled together on trial one and trial two with 8 total words correctly recalled on trial one $(t=8)$ and 9 total words correctly recalled on the trial two $(t+1=9)$. If there were 4 words in common across both trials (but only one subjective cluster), the subjective clustering expected score would be calculated using: $\mathrm{c}=4$ ( 4 words recalled on both trial $1 \&$ trial 2 ), $\mathrm{h}=8$ as trial 1 had 8 total correct words recalled, $\mathrm{k}=9$ as trial 2 had 9 total correct words recalled: $2(4) *(4-1) /(8 * 9)=0.333$. This result is then inserted into the $\mathrm{CA}$ subjective clustering formula of observed subjective clustering (car/lettuce, subjective observed score of 1) minus expected subjective clustering or $[1-0.333]=0.667$, yielding a subjective clustering score of 0.667 for trial 1 to trial 2. A higher number demonstrates greater frequency of subjective clustering.

\section{Chance adjusted (CA) serial clustering individual trials}

Serial clustering encompasses recalling items in the order in which they were presented. The serial position effect (Young, Hakes, \& Hicks, 1965) is demonstrated by a tendency to recall more items from the first (i.e., primacy) and last (i.e., recency) portions of a word list. On the CVLT-II, a serial recall strategy is an extension of the serial position effect as it involves grouping items in the order in which they were presented. For serial cluster scoring, one point was given each time two correct items from the list are recalled in the same order in which they were presented. For example, successive recall of the second and third words would yield a serial forward order score of one. We also scored serial clusters backward with one point given every time a correct target word immediately followed another correct target word in reverse order. 
The bidirectional serial clustering observed score encompasses a summation of observed forward (F) serial clustering and observed backward (B) serial clustering. The CA serial clustering score is illustrated in Equation 3:

$$
\begin{aligned}
& \text { Eq. 3. (Observed F + B Serial Cli) }- \text { Expected F } \\
& +\mathrm{B} \text { Serial Cl } i=\frac{[(c-1)]}{15}
\end{aligned}
$$

where " $\mathrm{i}$ " is represents a given trial and "c" is the number of correctly recalled items for the trial. The CA serial score thereby reflects observed bidirectional serial clustering minus expected bidirectional serial clustering.

\section{Statistical Analysis}

Consistent with previous work (Geary et al., 2010), data from each individual were fitted to a power function (Eq. 4). The power function, which is commonly applied in the behavioral learning literature (Anderson, 1982; Logan, 1998), was done by applying a two-parameter power function and calculating the best-fit line. The primary dependent measure for this analysis was total number of correctly recalled items per trial. This function was applied to data from each participant. We extracted the y-intercept (represented by $y$ in Eq. 4), which equates to the location at which the best-fit line crosses the y-axis, and slope (represented by $b$ in Eq. 4) which reflects how quickly learning is accomplished and/or the position at which the line becomes asymptotic. Unlike the CVLT-II learning trials $1-5$ slope which reflects a least squares linear regression, the power function allows for characterization of the rate of change (exponential growth).

$$
\text { Eq. 4. } \quad y=a x^{b}
$$

Correlations and regression analyses were used to evaluate the extent to which each of the clustering strategies predicted the rate of learning, the primary outcome measure, in patients and controls separately. First, Pearson's correlations were conducted to evaluate the unadjusted relationship between the three CA clustering strategies and overall rate of learning. Next, stepwise regression analyses were conducted to assess the extent to which each strategy contributed unique variance to overall learning rate.

\section{RESULTS}

Consistent with our prior reported observations (Geary et al., 2010), groups differed on performance on the initial learning trial of the CVLT-II $(p<.05)$. This relationship is shown in Figure 1a. Table 3 details performance on CVLT-II variables. Groups did not differ significantly on total learning or delayed memory scores or ListB recall (all $p$ 's $>.05$ ). Groups did differ on average CA semantic clustering across five trials $(p<.05)$.

Pearson's correlations were conducted to evaluate the unadjusted relationship between the three CA clustering (a) Raw Trial-Wise Recall

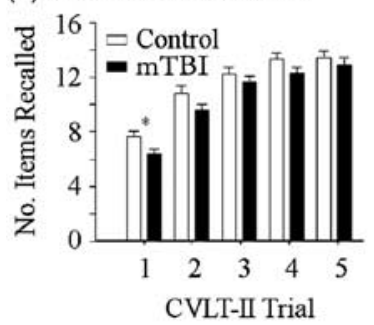

(c) Adjusted Subjective Clusters

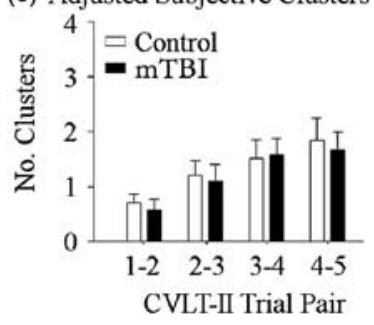

(b) Adjusted Semantic Clusters

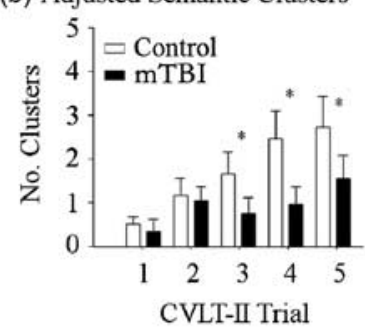

(d) Adjusted Serial Clusters

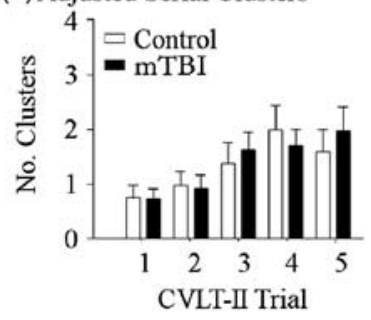

Fig. 1. a: CVLT-II raw recall findings across trials one through five for controls and patients with mTBI. Statistically significant difference between groups was only observed on the first learning trial. b: Chance adjusted semantic clusters across trials one through five for control and mTBI participants. Statistically significant differences between groups were observed on trials three through five. c: Chance adjusted subjective clusters scores across trials for control and mTBI participants. No statistically significant differences between groups were observed across trials. $\mathrm{d}$ : Chance adjusted serial clusters across trials one through five for control and mTBI participants. No statistically significant differences between groups were observed across trials.

strategies and overall rate of learning. For control participants, average CA semantic $(r=0.566 ; p<.01)$ and average CA subjective $(r=0.565 ; p<.01)$ clustering was related to overall learning rate. For mTBI, only CA serial clustering was related to overall learning rate $(r=0.432 ; p<.01)$.

To test our primary hypothesis that clustering strategy could explain learning rate on the CVLT-II, a stepwise linear regression analysis was undertaken by group entering the average five-trial CA semantic clustering score, average five-trial bidirectional CA serial clustering score, and average four CA subjective clustering scores, as predictors of rate of overall learning. These analyses revealed that for the control participants, average CA semantic clustering score $(\beta=1.17 ; t(25)=6.45 ; p<.001)$ and average $\mathrm{CA}$ serial clustering score $(\beta=0.82 ; t(25)=4.53 ; p<.001)$ were significant predictors of overall rate of learning $\left(R^{2}=0.63\right.$; $F(2,25)=20.980 ; p<.001)$ accounting for $32 \%$ and $31 \%$, respectively, of the variance in overall learning rate. For mTBI participants, only the average CA serial clustering score $(\beta=0.43 ; t(33)=2.75 ; p<.01)$ was a significant predictor of learning rate $(F(1,33)=7.58 ; p<.01)$ accounting for $19 \%$ of the variance.

To better understand differences in strategy use on the CVLT-II, we conducted three separate post hoc mixed factor analyses of variance (i.e., one per clustering strategy). 
Table 3. Raw scores of CVLT-II performance

\begin{tabular}{|c|c|c|c|c|c|c|c|}
\hline & \multicolumn{2}{|c|}{ Control $(N=28)$} & \multicolumn{2}{|c|}{$\operatorname{mTBI}(N=35)$} & \multirow{2}{*}{$t$ value } & \multirow{2}{*}{$p$ value } & \multirow{2}{*}{$\eta^{2}$} \\
\hline & Mean & $S D$ & Mean & $S D$ & & & \\
\hline Trial 1 Raw & 7.64 & 2.04 & 6.40 & 1.79 & 2.576 & 0.012 & 0.098 \\
\hline Trial 2 Raw & 10.79 & 2.62 & 9.57 & 2.67 & 1.810 & 0.075 & 0.051 \\
\hline Trial 3 Raw & 12.21 & 2.47 & 11.66 & 2.46 & 0.892 & 0.376 & 0.013 \\
\hline Trial 4 Raw & 13.29 & 2.32 & 12.26 & 2.60 & 1.633 & 0.108 & 0.042 \\
\hline Trial 5 Raw & 13.46 & 2.01 & 12.94 & 2.48 & 0.900 & 0.372 & 0.013 \\
\hline Total Trials $1-5$ Raw & 57.39 & 9.61 & 52.83 & 10.26 & 1.804 & 0.076 & 0.051 \\
\hline List B Raw & 6.93 & 2.72 & 6.34 & 2.26 & 0.933 & 0.354 & 0.014 \\
\hline Short-Free Recall Raw & 12.04 & 3.43 & 11.29 & 2.81 & 0.954 & 0.344 & 0.015 \\
\hline Short-Cued Recall Raw & 12.46 & 2.55 & 11.80 & 2.87 & 0.960 & 0.341 & 0.015 \\
\hline Long-Free Recall Raw & 12.43 & 3.27 & 11.46 & 2.89 & 1.249 & 0.216 & 0.025 \\
\hline Long-Cued Recall Raw & 13.18 & 2.34 & 12.11 & 2.91 & 1.571 & 0.121 & 0.039 \\
\hline Recognition Hits Raw & 15.14 & 1.04 & 14.40 & 1.82 & 1.921 & 0.059 & 0.057 \\
\hline False Positive Hits Raw & 2.11 & 3.99 & 2.20 & 2.63 & -0.111 & 0.912 & 0.000 \\
\hline Discrimination Raw & 3.32 & 0.75 & 2.99 & 0.66 & 1.873 & 0.066 & 0.054 \\
\hline Forced Choice Raw & 16.00 & 0.00 & 15.97 & 0.18 & 0.878 & 0.384 & 0.014 \\
\hline Total Intrusions & 1.86 & 2.24 & 2.11 & 1.95 & 0.237 & 0.628 & 0.004 \\
\hline Average Chance Adjusted Semantic Clustering & 1.70 & 2.09 & 0.73 & 1.14 & 2.331 & 0.023 & 0.087 \\
\hline Average Chance Adjusted Serial Clustering & 1.36 & 1.32 & 1.42 & 1.29 & -0.186 & 0.853 & 0.001 \\
\hline Average Chance Adjusted Subjective Clustering & 1.30 & 1.06 & 1.23 & 1.30 & 0.245 & 0.807 & 0.001 \\
\hline
\end{tabular}

We used the strategy score for each trial as the withinsubject factor (e.g., (1)CA semantic clustering score on five trials, (2)CA serial clustering score on five trials; (3)four CA subjective clustering scores: trial 1 to trial 2; trial 2 to trial 3; trial 3 to trial 4; trial 4 to trial 5) and Group (control, mTBI) as the between-subject factor. As shown in Figure 1b-d, there was no significant group by trial interaction effect for any clustering strategy or significant trial by trial differences between groups on CA subjective (Figure 1c) or CA serial clustering (Figure 1d) variables. However, these analyses revealed a significant between group effect with control participants using more CA semantic clusters compared to mTBI participants $F(1,61)=5.994 ; p<.001 ; \eta^{2}=0.091$. Table 4 details CA semantic clustering by group for each learning trial.

Although we did not include ListB in our primary learning analyses as it is difficult to reliably analyze strategy use in only one presentation and ListB shares semantic categories

Table 4. CVLT-II semantic clustering chance adjusted

\begin{tabular}{lccllcr}
\hline \hline & \multicolumn{2}{c}{ Control $(N=28)$} & & \multicolumn{2}{c}{$\operatorname{mTBI}(N=35)$} \\
\cline { 2 - 3 } & Mean & $S D$ & & Mean & $S D$ & \\
\hline Trial 1 & 0.47 & 1.01 & & 0.29 & 1.54 & \\
Trial 2 & 1.12 & 2.13 & & 1.02 & 1.86 & \\
Trial 3 & 1.64 & 2.69 & & 0.75 & 1.96 & $*$ \\
Trial 4 & 2.45 & 3.37 & & 1.03 & 2.35 & $*$ \\
Trial 5 & 2.94 & 3.48 & & 1.35 & 2.64 & $*$ \\
\hline \hline
\end{tabular}

Note. ${ }^{*} p<0.05$. with ListA (i.e., proactive interference effects), we did examine transfer of strategy effects by comparison of Trial 1 of ListA and ListB. Consistent with our previous work, our groups did not differ on total raw recall on ListB (Geary et al., 2010) and there were no between group differences or interaction effects. However, to examine potential transfer of strategy (DeRosa, Doane, \& Russell, 1970), we conducted post hoc stepwise regression analyses that demonstrated semantic clustering predicted $24 \%$ of the variance of ListB recall for controls only $(\beta=0.49 ; t(27)=3.75 ; p<.001)$. For mTBI, only serial clustering was a significant predictor of ListB recall $(\beta=0.36 ; t(33)=2.22 ; p<.05)$.

Recalling that our prior finding (Geary et al., 2010) was of a relationship of diminished recall on the first recall trial, we also conducted a post hoc examination of recall consistency across trials. This analysis revealed less consistency in recall in mTBI relative to controls from trial 1 to trial 2 , $t(61)=2.130, p=0.037$, but not on the remaining trials. Table 5 details these analyses.

Table 5. Recall consistency of Recall Across Trials

\begin{tabular}{|c|c|c|c|c|}
\hline & \multicolumn{2}{|c|}{ Control $(N=28)$} & \multicolumn{2}{|c|}{$\operatorname{mTBI}(N=35)$} \\
\hline & Mean & $S D$ & Mean & $S D$ \\
\hline Words Recalled T1-T2 & 6.32 & 2.37 & 5.14 & 2.02 \\
\hline Words Recalled T2-T3 & 9.14 & 2.97 & 7.77 & 3.01 \\
\hline Words Recalled T3-T4 & 11.00 & 3.14 & 9.83 & 2.88 \\
\hline Words Recalled T4-T5 & 11.68 & 3.02 & 10.69 & 3.11 \\
\hline
\end{tabular}

Note. $* p<0.05$ 


\section{DISCUSSION}

The current study serves to characterize the mechanisms that underlie reductions in rate of verbal learning in mTBI (Geary et al., 2010). To our knowledge, this is the first study to examine verbal learning strategy use within and across trials in a mTBI sample who achieved comparable total learning and memory scores relative to control participants. This approach is consistent with recent interest examining qualitative aspects of learning and memory performance, such as strategy use (Baldo, Delis, Kramer, \& Shimamura, 2002; Millis \& Ricker, 1994; Nolin, 2006; Schefft et al., 2008). Semantic and subjective strategy formation and implementation are considered qualitative aspects of learning and memory performance. Such behaviors fall under the category of executive functions (Alexander \& Stuss, 2006; Matsui et al., 2008) reflective of active engagement of self-generated or internally driven reasoning skill. Semantic clustering arguably represents the most efficient and highest-order organization strategy to facilitate learning (Becker \& Lim, 2003). Given the evidence of frontal lobe dysfunction and reduced strategy use in TBI of greater severity (Levine et al., 1998; Millis \& Ricker, 1994; Schefft et al., 2008; Strangman et al., 2008), we questioned if diminished internally derived meta-cognitive strategy use could explain decreased rate of learning across trials in a mTBI sample. Our present findings are supportive of the hypothesis that mTBI participants are under-utilizing semantic clustering relative to control participants. In the context of comparable total immediate recall and delayed memory scores, control participants use semantic clustering whereas the mTBI do not to a similar degree.

The frontal lobe's involvement in executive functions such as strategic processes of learning and memory is well supported (Alexander, Stuss, \& Fansabedian, 2003; Alexander et al., 2009; Baldo et al., 2002; Cabeza \& Nyberg, 2000; Turner, Cipolotti, Yousry, \& Shallice, 2007; Turriziani, Smirni, Oliveri, Semenza, \& Cipolott, 2010). We have previously reported no significant group differences between control and mTBI participants on administered measures of executive functioning (Geary et al., 2010; Kraus et al., 2007). In retrospect, these previous reports may not have been sufficient to conclude that subtle executive deficits do not persist following mTBI. We undertook the current analysis with the speculation that perhaps our executive function measures were not sensitive to detect subtle but diffuse deficits that may be experienced following mTBI (Cicerone et al., 2006).

Traditionally, varied and overlapping skills believed dependent on prefrontal cortex are grouped under the executive function rubric (Stuss \& Levine, 2002). Executive functions can be conceptualized as a hierarchy of cognitive processes with meta-cognitive processes such as those related to internally derived strategy use at the apex. In our larger battery, our executive function measures (set-shifting, response-inhibition, sustained attention) (Kraus et al., 2007) share a common feature of an externally facilitated structure through the form of verbal instruction, visual stimulus, or visual feedback. In this way, these measures provide overt passive "structure" to the tasks. It may be that examining individualized aspects of performance in mTBI may increase the sensitivity of assessment (Cicerone et al., 2006; Stuss \& Levine, 2002) and capture internally derived executive functions that may be more diffusely represented such as strategy use (Cicerone et al., 2006).

Meta-cognitive functions also includes the awareness that strategy use facilitates learning/recall on a word-list and then using that strategy in another word-list (Ellis, 1965). In the CVLT-II, transfer of learning strategy is likely evident when semantic clustering is used both during ListA learning trials and on the single presentation of ListB (DeRosa et al., 1970). Our groups did not differ on total raw recall on ListB and there were no between group difference or interaction effect evident on repeated ANOVA comparing ListA trial 1 to ListB raw recall performance (Geary et al., 2010). ListB consists of 16 items from four semantic categories, two categories overlap with categories on ListA. Despite proactive interference effects which are greatest among words from shared semantic categories (Delis et al., 2000b), post hoc stepwise regression found that semantic clustering predicted ListB recall for controls, but not for mTBI. This finding offers additional support that the mTBI participants exhibit deficient semantic strategy use as they under-use the semantic clustering strategy with a novel word list.

Unlike semantic clustering, serial clustering does not involve actively restructuring information as it is presented. Rather, serial clustering is externally facilitated as it embodies recalling items in the order in which they are presented. An over-reliance on serial clustering, at the expense of semantic clustering, in other neurological populations has been demonstrated to negatively correlate with overall recall (Delis et al., 1988; Gsottschneider et al., 2010; Jefferies et al., 2008; Ranjith et al., 2010). Our present findings are consistent with our hypothesis that mTBI participants use a less efficient serial strategy relative to controls. For mTBI participants, averaged CA serial clustering was the only significant predictor of learning rate.

As diffuse or traumatic axonal injury is the most frequent neuropathologic observation following mTBI of all etiologies, it has been speculated that disrupted connection between frontal-subcortical networks could explain deficiencies in cognitive performance (Becker \& Lim, 2003; Ghajar, Ivry, \& The Cognitive Neurobiological Consortium, 2008; Hartikainen et al., 2010; Zappalá \& Trexler, 1992). This hypothesis was recently examined using functional magnetic resonance imaging in TBI participants (mild-severe) during performance of a list-learning paradigm (Strangman et al., 2008). Participants were imaged under three listlearning conditions, two of which involved semantically related word-lists. On the final "directed" condition, participants were instructed on the use of a semantic clustering strategy. Findings revealed that during the directed semantic clustering condition, both TBI and control groups displayed improvements in recall, but that controls demonstrated 
increased coupling with activation observed in dorsolateral prefrontal cortex (DLPFC) and angular gyrus (AG), while the TBI participants did not. These findings were interpreted as indications of variable disruptions along the superior longitudinal fasciculus (SLF) connecting angular gyrus and DLPFC. The authors speculated that while the TBI participants did not engage the more efficient DLPFC-AG network, they still experienced improvements in learning by a separate processing network. These findings have particular relevance given prior report of a relationship between integrity of the SLF assessed via diffusion tensor imaging and behavior (Bendlin et al., 2008; Geary et al., 2010; Kinnunen et al., 2011; Mayer et al., 2009; Sidaros et al., 2009). From these works, the possibility is raised that dysfunction of the SLF in mTBI may underlie deficient meta-cognitive strategy use and explain over-reliance on more externally derived strategy use.

The use of more externally driven strategies or application of variable strategies may result in inconsistent patterns of recall. Indeed, list recall in moderate-severe TBI has been suggestive of a disorganized haphazard learning style coupled with an increased reliance on serial clustering (Deluca, Schultheis, Madigan, Christodoulou, \& Averill, 2000; Millis \& Ricker, 1994). Recalling that our prior work focused on early learning inefficiency (Geary et al., 2010), our finding of less consistent recall from trial 1 to trial 2 may suggest that the mTBI participants are responding to the second trial as if it were a novel list versus a repeated presentation (Delis et al., 2000a) or possibly reflective of diminished attention (DeJong $\&$ Donders, 2010). This has also been offered as a theory to explain behavior in patients with frontal lobe dysexecutive syndrome (Roofeh et al., 2006; Stuss \& Alexander, 2007). We also considered that our mTBI participants might commit more intrusion errors reflective of reduced self-monitoring as has been offered by others (Busch, McBride, Curtiss, $\&$ Vanderploeg, 2005), but this was not the case $(p>.05)$, suggesting no source memory problems.

\section{Study Limitations}

In any TBI study, a primary concern is the inclusion of participants with a history of mTBI without witness confirmation of LOC or PTA. While our inclusion criteria was biased against inclusion of those with potentially greater severity of injury, given the reliance on retrospective selfreport, it is possible that some of these individuals $(\mathrm{N}=14$ without witness-confirmed LOC or PTA) either did not sustain a TBI or sustained a TBI of greater than mild severity. Additionally, there is always concern with lifetime history and inclusion of participants with multiple TBIs. In fact, 12 of the TBI participants in this study reported a history of multiple mTBI. Primary CVLT-II trials 1-5, total learning, ListB and delayed memory analyses conducted with and without these participants demonstrated no change in the previously published findings (Geary et al., 2010). However, while comparisons of single versus multiple mTBI participants detected no significant differences between the TBI groups on variables of interest, the inclusion of individuals with multiple injuries raises the possibility that findings could be driven, in part, by changes attributable to multiple mild injuries as has been suggested by others (Weber, 2007). As such, future studies should be undertaken examining strategy use in a large group of patients with multiple mTBI so that number of TBIs can be examined directly. Furthermore, future studies would benefit by the collection of objective data on the duration of LOC and objective measurements of PTA for each injury. A prospective, longitudinal investigation of acute TBI course and recovery would achieve such aims.

We did not collect any data regarding the functional significance of the initial learning deficiency or ask any questions particularly relevant to meta-cognitive strategy use (e.g., "do you find it harder to organize information during your day-to-day?"). Future studies comparing strategy use and learning performance to more specific outcome variables would prove especially informative.

Despite these limitations, the clinical significance of reduced meta-cognitive strategy use in mTBI participants warrants further exploration. Notably, our groups did not differ on standard measures of executive function, which some suggest may not be sensitive to detect the subtle diffuse deficits following mTBI (Cicerone et al., 2006; Stuss \& Levine, 2002). Given the continued debate regarding persisting cognitive deficits following mTBI and the issues regarding the ecological validity and sensitivity of neuropsychological assessment to detect persisting cognitive changes in patients with a history of mTBI (Alexander, 1995; Iverson, 2010; Satz et al., 1999; Silver, 2000), this study endeavored to elaborate on the individualized learning strategies of mTBI participants. Specifically, while chronic memory dysfunction is not supported in the mTBI literature, the issue may be one of what constitutes "memory" as standardly interpreted in neuropsychological evaluations. Perhaps the persisting learning and memory difficulties reported by some mTBI patients are related to reduced usage of internally driven strategies that facilitate learning and enhance recall. That mTBI participants use less semantic clusters relative to controls and use serial strategies is compelling especially given the comparable total learning (trials 1-5) score. Adopting a serial recall strategy versus a semantic strategy could require TBI participants to use other cognitive processes (Strangman et al., 2008) to achieve comparable total learning scores. Given that strategy training has demonstrated improvements in learning and memory (Basso, Lowery, Ghormley, Combs, \& Johnson, 2006; Fiszdon et al., 2006; O’Brien, Chiaravalloti, ArangoLasprilla, Lengenfelder, \& DeLuca, 2007; Schefft et al., 2008), these findings have translation value in offering that mTBI patients be given recommendations such as consideration of strategy use when learning information to potentially remediate learning inefficiencies.

\section{ACKNOWLEDGMENTS}

This work is supported in part by NIH grant K23 MH068787 (MFK) and T32-MH067631 (EKG) from the National Institute of Mental 
Health, the Marshall Goldberg Traumatic Brain Injury Fund, NICHD/ORWH grant K12HD055892 (LHR), and a grant from the Department of Defense/Congressionally Directed Medical Research Program grant PT 075675 . The contents of this study are solely the responsibility of the authors and do not necessarily represent the official views of the University of Illinois at Chicago, Department of Defense, Congressionally Directed Medical Research Program, Department of the Army, National Institute of Child Health and Human Development, the National Institutes of Health, or the National Institute of Mental Health. Special thanks to Sarung Kashyap for his contribution in the preparation of the raw data for these analyses. The authors have no financial and/or relationship conflicts of interest to disclose.

\section{REFERENCES}

Alexander, M. (1995). Mild traumatic brain injury: Pathophysiology, natural history, and clinical management. Neurology, 45, 1253-1260.

Alexander, M., \& Stuss, D. (2006). Frontal injury: Impairments of fundamental processes lead to functional consequences. Journal of the International Neuropsychological Society, 12(2), 192-193.

Alexander, M., Stuss, D., \& Fansabedian, N. (2003). California Verbal Learning Test: Performance by patients with focal frontal and non-frontal lesions. Brain, 126(Pt 6), 1493-1503.

Alexander, M., Stuss, D., \& Gillingham, S. (2009). Impaired list learning is not a general property of frontal lesions. Journal of Cognitive Neuroscience, 21(7), 1422-1434.

American Congress on Rehabilitation Medicine. (1993). Definition of mild traumatic brain injury. Journal of Head Trauma Rehabilitation, 8, 86-87.

Anderson, J. (1982). Acquisition of a cognitive skill. Psychological Review, 89, 369-406.

Baldo, J., Delis, D., Kramer, J., \& Shimamura, A. (2002). Memory performance on the California Verbal Learning Test-II: Findings from patients with focal frontal lesions. Journal of the International Neuropsychological Society, 8(4), 539-546.

Basso, M., Lowery, N., Ghormley, C., Combs, D., \& Johnson, J. (2006). Self-generated learning in people with multiple sclerosis. Journal of the International Neuropsychological Society, 12(5), 640-648.

Becker, S., \& Lim, J. (2003). A computational model of prefrontal control in free recall: Strategic memory use in the California Verbal Learning Task. Journal of Cognitive Neuroscience, 15(6), 821-832.

Bendlin, B., Ries, M., Lazar, M., Alexander, A., Dempsey, R., Rowley, H., ... Johnson, S. (2008). Longitudinal changes in patients with traumatic brain injury assessed with diffusion-tensor and volumetric imaging. Neuroimage, 42(2), 503-514.

Benedictus, M., Spikman, J., \& van der Naalt, J. (2010). Cognitive and behavioral impairment in traumatic brain injury related to outcome and return to work. Archives of Physical Medicine and Rehabilitation, 91(9), 1436-1441.

Binder, L., Rohling, M., \& Larrabee, G. (1997). A review of mild head trauma. Part I: Meta-analytic review of neuropsychological studies. Journal of Clinical and Experimental Neuropsychology, 19(3), 421-431.

Bruce, J., \& Echemendia, R. (2003). Delayed-onset deficits in verbal encoding strategies among patients with mild traumatic brain injury. Neuropsychology, 17(4), 622-629.

Busch, R., McBride, A., Curtiss, G., \& Vanderploeg, R. (2005). The components of executive functioning in traumatic brain injury. Journal of Clinical and Experimental Neuropsychology, 27(8), 1022-1032.
Cabeza, R., \& Nyberg, L. (2000). Neural bases of learning and memory: Functional neuroimaging evidence. Current Opinion in Neurology, 13(4), 415-421.

Cassidy, J., Carroll, L., Peloso, P., Borg, J., von Holst, H., Holm, L., ... WHO Collaborating Centre Task Force on Mild Traumatic Brain Injury. (2004). WHO Collaborating Centre Task Force on Mild Traumatic Brain Injury. Incidence, risk factors and prevention of mild traumatic brain injury: Results of the WHO Collaborating Centre Task Force on Mild Traumatic Brain Injury. Journal of Rehabilitation Medicine, 43(Suppl), 28-60.

Chan, A., Kwoka, I., Chiub, H., Lamb, L., Pangb, A., \& Chow, L. (2000). Memory and organizational strategies in chronic and acute schizophrenic patients. Schizophrenia Research, 41(3), 431-445.

Cicerone, K., \& Kalmar, K. (1995). Persistent postconcussion syndrome: The structure of subjective complaints after mild traumatic brain injury. The Journal of Head Trauma Rehabilitation, 10(3), 1-17.

Cicerone, K., Levin, H., Malec, J., Stuss, D., \& Whyte, J. (2006). Cognitive rehabilitation interventions for executive function: Moving from bench to bedside in patients with traumatic brain injury. Journal of Cognitive Neuroscience, 1(7), 1212-1222.

DeJong, J., \& Donders, J. (2010). Cluster subtypes on the California Verbal Learning Test-Second Edition (CVLT-II) in a traumatic brain injury sample. Journal of Clinical and Experimental Neuropsychology, 32(9), 953-960.

Delis, D., Fine, E., Stricker, J., Houston, W., Wetter, S., Cobell, K., ... Bondi, M. (2010). Comparison of the traditional recall-based versus a new list-based method for computing semantic clustering on the California Verbal Learning Test: Evidence from Alzheimer's disease. The Clinical Neuropsychologist, 24(1), 70-79.

Delis, D., Freeland, J., Kramer, J., \& Kaplan, E. (1988). Integrating clinical assessment with cognitive neuroscience: Construct validation of the California Verbal Learning Test. Journal of Consulting and Clinical Psychology, 56(1), 123-130.

Delis, D., Kramer, J., Kaplan, E., \& Obers, B. (2000a). California Verbal Learning Test-examiners manual (Second-Adult Version ed.). San Antonio, TX: The Psychological Corporation.

Delis, D., Kramer, J., Kaplan, E., \& Obers, B. (2000b). California Verbal Learning Test-II (2nd ed.). San Antonio, TX: Psychological Corporation.

Deluca, J., Schultheis, M., Madigan, N., Christodoulou, C., \& Averill, A. (2000). Acquisition versus retrieval deficits in traumatic brain injury: Implications for memory rehabilitation. Archives of Physical Medicine and Rehabilitation, 81, 1327-1333.

DeRosa, D., Doane, D., \& Russell, B. (1970). The influence of first-list organization upon second-list free-recall learning. Journal of Verbal Learning and Verbal Behavior, 9(3), 269-273.

Dikmen, S., Corrigan, J., Levin, H., Machamer, J., Stiers, W., \& Weisskopf, M. (2009). Cognitive outcome following traumatic brain injury. The Journal of Head Trauma Rehabilitation, 24(6), 430-438.

Ellis, H. (1965). The transfer of learning. New York: MacMillan.

Ettenhofer, M., \& Abeles, N. (2009). The significance of mild traumatic brain injury to cognition and self-reported symptoms in long-term recovery from injury. Journal of Clinical and Experimental Neuropsychology, 31(3), 363-372.

Fiszdon, J., McClough, J., Silverstein, S., Bell, M., Jaramillo, J., \& Smith, T. (2006). Learning potential as a predictor of readiness for psychosocial rehabilitation in schizophrenia. Psychiatry Research, 143(2-3), 159-166. 
Geary, E., Kraus, M., Pliskin, N., \& Little, D. (2010). Verbal learning differences in chronic mild traumatic brain injury. Journal of the International Neuropsychological Society, 16, 506-516.

Ghajar, J., \& Ivry, R., The Cognitive Neurobiological Consortium (2008). The predictive brain state: Timing deficiency in traumatic brain injury? Neurorehabilitation and Neural Repair, 22, 217-227.

Gongvatana, A., Woods, S., Taylor, M., Vigil, O., Grant, I., \& Group, T.H. (2007). Semantic clustering inefficiency in HIV associated dementia. The Journal of Neuropsychiatry and Clinical Neurosciences, 19, 36-42.

Gsottschneider, A., Keller, Z., Pitschel-Walz, G., Froböse, T., Bäuml, J., \& Jahn, T. (2010). The role of encoding strategies in the verbal memory performance in patients with schizophrenia. Journal of Neuropsychology, [Epub ahead of print].

Hartikainen, K., Waljas, M., Isoviita, T., Dastidar, P., Liimatainen, S., Solbakk, A., ... Ohman, J. (2010). Persistent symptoms in mild to moderate traumatic brain injury associated with executive dysfunction. Journal of Clinical and Experimental Neuropsychology, 32(7), 767-774.

Iverson, G. (2005). Outcome from mild traumatic brain injury. Current Opinion in Psychiatry, 18, 301-317.

Iverson, G. (2010). Mild traumatic brain injury meta-analyses can obscure individual differences. Brain Injury, 24(10), 1246-1255.

Jefferies, E., Hoffman, P., Jones, R., \& Ralph, M. (2008). The impact of semantic impairment on verbal short-term memory in stroke aphasia and semantic dementia: A comparative study. Journal of Memory and Language, 58(1), 66-87.

Kinnunen, K., Greenwood, R., Powell, J., Leech, R., Hawkins, P., Bonnelle, V., ... Sharp, D. (2011). White matter damage and cognitive impairment after traumatic brain injury. Brain, 134(Pt 2), 449-463.

Kraus, M., Susmaras, T., Caughlin, B., Walker, C., Sweeney, J., \& Little, D. (2007). White matter integrity and cognition in chronic traumatic brain injury: A diffusion tensor imaging study. Brain, 130, 2508-2519.

Levin, H. (1992). Neurobehavioral Recovery. Journal of Neurotrauma, 9, S359-S373.

Levine, B., Stuss, D., Milberg, W., Alexander, M., Schwartz, M., \& Macdonald, R. (1998). The effects of focal and diffuse brain damage on strategy application: Evidence from focal lesions, traumatic brain injury and normal aging. Journal of the International Neuropsychological Society, 4(3), 247-264.

Little, D., Kraus, M., Joseph, J., Geary, E., Susmaras, T., Zhou, X., ... Gorelick, P. (2010). Thalamic integrity underlies executive dysfunction in traumatic brain injury. Neurology, 74(7), 558-564.

Logan, G. (1998). Toward an instance theory of automatization. Psychological Review, 95, 492-527.

Luek, S. (1976). Spontaneous categorizers retain more than spontaneous alphabetizers. Memory \& Cognition, 4(5), 476-482.

Matsui, M., Suzuki, M., Zhou, S., Takahashi, T., Kawasaki, Y., Yuuki, H., ... Kurachi, M. (2008). The relationship between prefrontal brain volume and characteristics of memory strategy in schizophrenia spectrum disorders. Progress in Neuropsychopharmacology \& Biological Psychiatry, 32(8), 1854-1862.

Mayer, A., Mannell, M., Ling, J., Elgie, R., Gasparovic, C., Phillips, J., ... Yeo, R. (2009). Auditory orienting and inhibition of return in mild traumatic brain injury: A FMRI study. Human Brain Mapping, 30(12), 4152-4166.

Millis, S., \& Ricker, J. (1994). Verbal learning patterns in moderate and severe traumatic brain injury. Journal of Clinical and Experimental Neuropsychology, 16(4), 498-507.
Nolin, P. (2006). Executive memory dysfunctions following mild traumatic brain injury. The Journal of Head Trauma Rehabilitation, 21(1), 68-75.

O’Brien, A., Chiaravalloti, N., Arango-Lasprilla, J., Lengenfelder, J., \& DeLuca, J. (2007). An investigation of the differential effect of self-generation to improve learning and memory in multiple sclerosis and traumatic brain injury. Neuropsychological Rehabilitation, 17(3), 273-292.

Ponsford, J., Willmott, C., Rothwell, A., Cameron, P., Kelly, A., Nelms, R., ... Ng, K. (2000). Factors influencing outcome following mild traumatic brain injury in adults. Journal of the International Neuropsychological Society, 6, 568-579.

Ranjith, N., Mathuranath, P., Sharma, G., \& Alexander, A. (2010). Qualitative aspects of learning, recall, and recognition in dementia. Annals of Indian Academy of Neurology, 13(2), 117-122.

Ribeiro, F., Guerreiro, M., \& De Mendonça, A. (2007). Verbal learning and memory deficits in Mild Cognitive Impairment. Journal of Clinical and Experimental Neuropsychology, 29(2), 187-197.

Roofeh, D., Cottone, J., Burdick, K., Lencz, T., Gyato, K., Cervellione, K., ... Kumra, S. (2006). Deficits in memory strategy use are related to verbal memory impairments in adolescents with schizophrenia-spectrum disorders. Schizophrenia Research, 85(1-3), 201-212.

Satz, P., Alfano, M., Light, R., Morgenstern, H., Zaucha, K., Asarnow, R., ... Newton, S. (1999). Persistent post-concussive syndrome: A proposed methodology and literature review to determine the effects, if any, of mild head and other bodily injury. Journal of Clinical and Experimental Neuropsychology, 21(5), 620-628.

Schefft, B., Dulay, M., \& Fargo, J. (2008). The use of a selfgeneration memory encoding strategy to improve verbal memory and learning in patients with traumatic brain injury. Applied Neuropsychology, 15(1), 61-68.

Schretlen, D., \& Shapiro, A. (2003). A quantitative review of the effects of traumatic brain injury on cognitive functioning. International Review of Psychiatry (Abingdon, England), 15(4), 341-349.

Schweizer, T., Alexander, M., Gillingham, S., Cusimano, M., \& Stuss, D. (2010). Lateralized cerebellar contributions to word generation: A phonemic and semantic fluency study. Behavioural Neurology, 23(1-2), 31-37.

Sidaros, A., Skimminge, A., Liptrot, M., Sidaros, K., Engberg, A., Herning, M., ... Rostrup, E. (2009). Long-term global and regional brain volume changes following severe traumatic brain injury: A longitudinal study with clinical correlates. Neuroimage, 44(1), $1-8$

Silver, C. (2000). Ecological validity of neuropsychological assessment in childhood traumatic brain injury. Journal of Head Trauma Rehabilitation, 15(4), 973-988.

Sternberg, R., \& Tulving, E. (1977). The measurement of subjective organization in free recall. Psychological Bulletin, 84, 539-556.

Strangman, G., O’Neil-Pirozzi, T., Goldstein, R., Kelkar, K., Katz, D., Burke, D., ... Glenn, M. (2008). Prediction of memory rehabilitation outcomes in traumatic brain injury by using functional magnetic resonance imaging. Archives of Physical Medicine and Rehabilitation, 89(5), 974-981.

Stricker, J., Brown, G., Wixted, J., Baldo, J., \& Delis, D. (2002). New semantic and serial clustering indices for the California Verbal Learning Test-Second Edition: Background, rationale, and formulae. Journal of the International Neuropsychological Society, 8(3), 425-435. 
Stuss, D., \& Alexander, M. (2007). Is there a dysexecutive syndrome? Philosophical Transactions of the Royal Society of London. Series B, Biological Sciences, 362(1481), 901-915.

Stuss, D., \& Levine, B. (2002). Adult clinical neuropsychology: Lessons from studies of the frontal lobes. Annual Review of Psychology, 53, 401-433.

Tagliaferri, F., Compagnone, C., Korsic, M., Servadei, F., \& Kraus, J. (2006). A systematic review of brain injury epidemiology in Europe. Acta Neurochirurgica, 148(3), 255-268.

Turner, M., Cipolotti, L., Yousry, T., \& Shallice, T. (2007). Qualitatively different memory impairments across frontal lobe subgroups. Neuropsychologia, 45(7), 1540-1552.

Turriziani, P., Smirni, D., Oliveri, M., Semenza, C., \& Cipolott, L. (2010). The role of the prefrontal cortex in familiarity and recollection processes during verbal and non-verbal recognition memory: An rTMS study. Neuroimage, 52(1), 348-357.

Weber, J. (2007). Experimental models of repetitive brain injuries. Progress in Brain Research, 161, 253-261.

West, L., Curtis, K., Greve, K., \& Bianchini, K. (2010). Memory in traumatic brain injury: The effects of injury severity and effort on the Wechsler Memory Scale-III. Journal of Neuropsychology, [Epub ahead of print].

Young, R., Hakes, D., \& Hicks, R. (1965). Effects of list length in the Ebbinghaus derived-list paradigm. Journal of Experimental Psychology, 70, 338-341.

Zappalá, G., \& Trexler, L. (1992). Quantitative and qualitative aspects of memory performance after minor head injury. Archives of Clinical Neuropsychology, 7(2), 145-154. 
Reproduced with permission of the copyright owner. Further reproduction prohibited without permission. 